\title{
Review
}

\section{e Functional Connectivity Alterations: Novel Therapy and Future Implications in Chronic Pain Management}

Stephen L. Thorp, MD¹, Thomas Suchy, MD², Nalini Vadivelu, MD², Erik M. Helander, MBBS ${ }^{3}$, Richard D. Urman, $\mathrm{MD}^{4}$, and Alan D. Kaye, MD, PhD ${ }^{3}$

From: ${ }^{1}$ Crystal Run Healthcare, Department of Pain Medicine, Middletown, NY; ${ }^{2}$ Yale-New Haven Hospital, Department of Anesthesiology, New Haven, CT; ${ }^{3}$ Department of Anesthesiology, LSU School of Medicine, New Orleans, LA; ${ }^{4}$ Department of Anesthesiology, Perioperative and Pain Medicine, Brigham and Women's Hospital, Boston, MA

Address Correspondence: Dr. Stephen Thorp, MD Crystal Run Healthcare Department of Pain Medicine 95 Crystal Run Road Middletown, NY E-mail:

sthorp@crystalrunhealthcare.com

Disclaimer: There was no external funding in the preparation of this manuscript. Conflict of interest: Each author certifies that he or she, or a member of his or her immediate family, has no commercial association (i.e., consultancies, stock ownership, equity interest, patent/licensing arrangements, etc.) that might pose a conflict of interest in connection with the submitted manuscript.

Manuscript received: 9-02-2017 Accepted for publication: o9-29-2017

Free full manuscript: www.painphysicianjournal.com
Background: Chronic pain is a major public health problem resulting in physical and emotional pain for individuals and families, loss of productivity, and an annual cost of billions of dollars. The lack of objective measures available to aid in diagnosis and evaluation of therapies for chronic pain continues to be a challenge for the clinician.

Objectives: Functional magnetic resonance imaging ( $\mathrm{fMRI}$ ) is an imaging technique that can establish regional areas of interest and examine synchronous neuronal activity in functionally related but anatomically distinct regions of the brain, known as functional connectivity.

Study Design: The present investigation examines changes in functional connectivity in 4 common pain syndromes: chronic back pain (CBP), fibromyalgia, diabetic neuropathy, and complex regional pain syndrome (CRPS).

Setting: This is a review of the current understanding of functional connectivity.

Methods: Utilizing functional imaging, patients with these conditions have been shown to have significant structural and functional differences when compared to healthy controls.

Results: Functional connectivity, therefore, has the potential to assist in diagnostic classification of different pain conditions, predict individual responses to specific therapeutic interventions, and serve as a gateway for personalized medicine. Indirect activation of brain activity can be seen by the blood flow to the brain at specific sites, with chronic pain patients having increased brain activity.

Limitations: The present investigation is limited in that few studies have examined this relatively new modality.

Conclusions: Knowing and observing the brain's activity as related to pain gives pain patients an opportunity to decrease pain-related brain activity and decrease severe chronic pain. This modality can be used along with interventional pain management techniques in order to provide optimum pain relief.

Key words: Functional connectivity, fMRI, chronic pain, chronic back pain, fibromyalgia, diabetic neuropathy, chronic regional pain syndrome

Pain Physician 2018; 21:E207-E214 hronic pain is a major public health problem, affecting approximately 100 million adults in the United States alone (1). The economic burden is equally staggering, estimated to be $\$ 560-635$ billion in the United States annually (2). Pain is a subjective sensation and is difficult to measure objectively, which leads to difficulty evaluating patients suffering with pain and evaluating their management. Identifying 


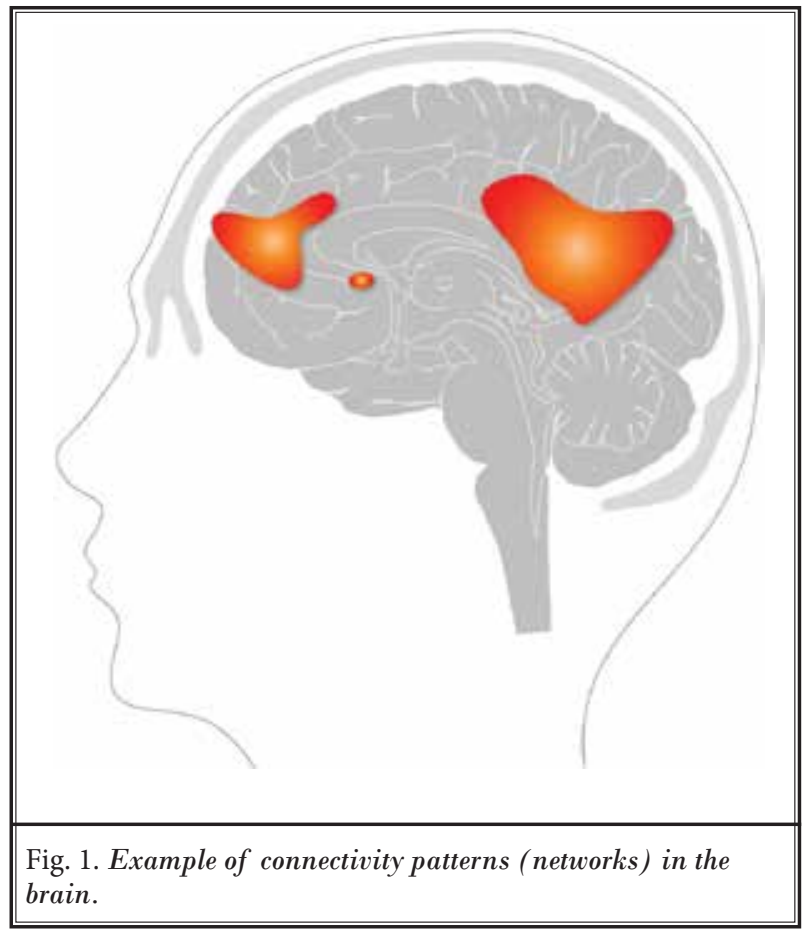

which patients will respond to particular medications or interventions, as well as which are likely to improve with more conservative measures, can dramatically improve the care of millions of patients (3). Objective measures for pain would aid these goals and improve the ability to diagnose accurately, evaluate efficacy of therapies, and eventually lead to better management.

Functional magnetic resonance imaging (fMRI) is a radiographic technique, which allows for imaging of functional activity in the brain. Regions of interest which involve numerous physical and emotional modalities have previously been identified with fMRI, including touch, motor activation, hearing, vision, memory, and anger. Similarly, several cortical and subcortical areas that are focused on pain perception and pain modulation have been identified. The primary and secondary somatosensory cortex, anterior cingulate cortex (ACC), and the insula (INS) are the regions most frequently activated by pain; however, other numerous regions have been shown to have increased activity in those suffering from chronic pain conditions as well (4).

fMRI can give information in the activation of brain areas as related to specific functions as well as functional connectivity (5). In fMRI, functional connectivity is emerging as an accepted method of studying global brain function in health and disease states. Connectivity refers to temporally synchronous neuronal activity in functionally related but anatomically distinct regions of the brain. Recent advances have suggested that neuronal function should be viewed somewhat like electroencephalography activity, since any functional activity in the brain is the end result of coordinated activity in multiple functionally related areas. Most pain activation studies have reported several activated regions, serving as an affirmation that neuronal activity is spatially widespread, linking many regions of the brain. Multiple areas of the brain are involved in pain perception and pain modulation. Thus, a focus of this review is on functional connectivity in 4 common pain syndromes: chronic back pain (CBP), fibromyalgia, diabetic neuropathy, and complex regional pain syndrome (CRPS).

\section{Functional Connectivity}

The human brain is composed of several anatomically distinct regions which are functionally related and connected through a highly organized network referred to as functional connectivity (6). Measuring connectivity is a method for non-invasively studying global neurological activity. Connectivity is established by observing changes in blood-oxygen-level dependent contrast (BOLD) in regional areas of interest. BOLD signals are generated by the variation in oxyhemoglobin to deoxyhemoglobin ratio induced by neuronal activity in the brain; hence, BOLD signaling is a qualitative measure of neuronal activity. Measuring BOLD signaling has become the best technique for measuring connectivity in the brain.

Based on studies in healthy volunteers, several connectivity patterns, referred to as networks, have been described in the literature. There are 10 networks that are well described and together cover more than $80 \%$ of the cerebral cortex. These networks include the default mode network (DMN) (Fig. 1), salience network, right and left executive control network, cerebellar network, sensorimotor network, auditory network, and 3 visual networks (medial, lateral, and occipital visual). The DMN has been studied most extensively by examining its anatomical connections, function at rest, influence on disease states, and how it is affected by pharmacological agents.

The DMN is linked to internal mentation of the brain and, in contrast to the other resting-state networks, shows increased activity during rest and decreased activity with task initiation. The regions associated with the DMN include the medial prefrontal cortex (mPFC), a set of regions located posteriorly midline (posterior cingulate cortex [PCC], precuneus, and inferior parietal lobe), 


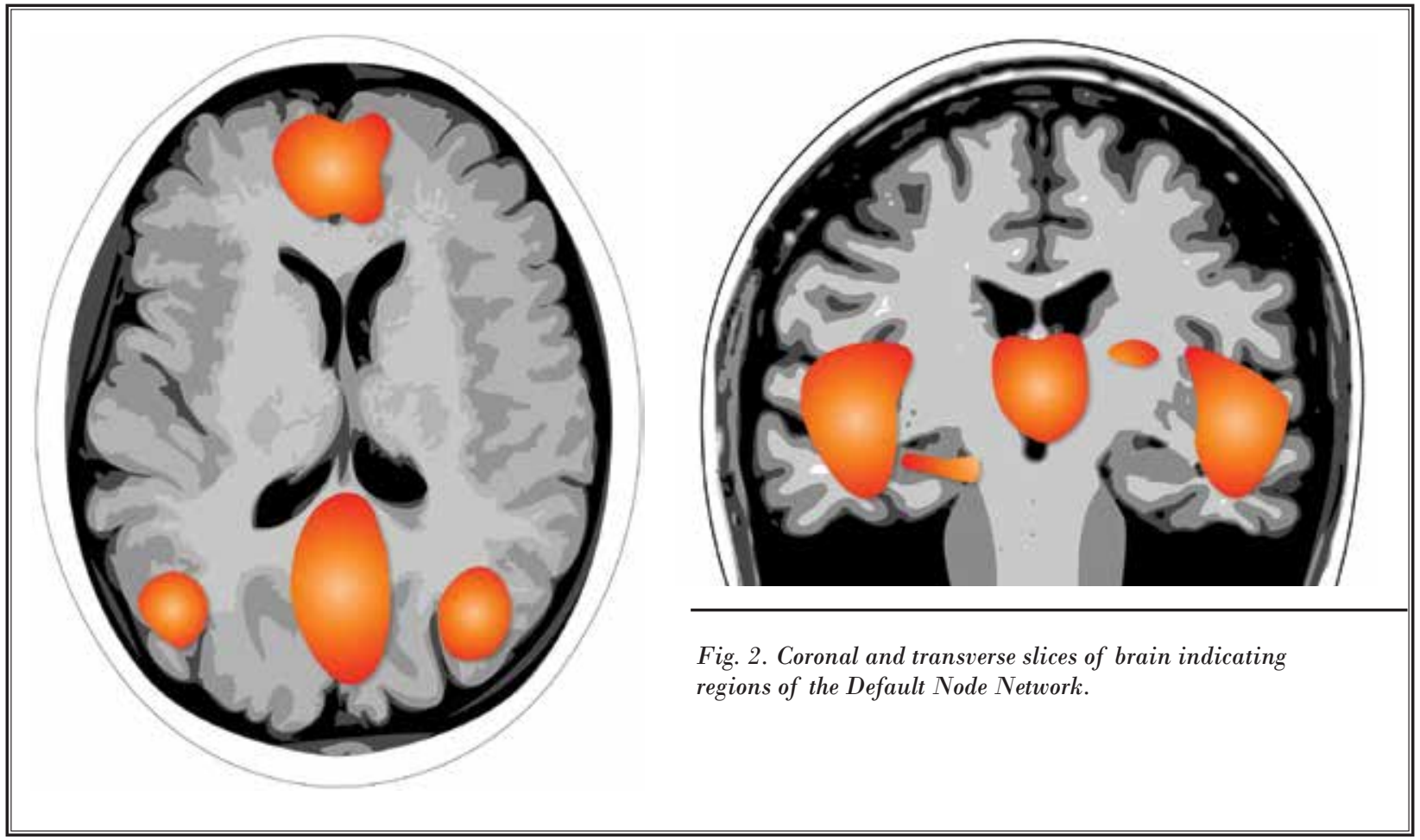

and the medial temporal cortex. Altered connectivity in the DMN has already been described in many neurological disorders, including schizophrenia, Alzheimer's disease, and attention deficit disorder. In recent years, studies examining the DMN have identified numerous alterations in functional activity in individuals suffering from chronic pain as well. In this regard, several studies have determined that there is correlation between the functional connectivity and pain symptoms in pain conditions, including CBP, fibromyalgia, CRPS, and diabetic neuropathy.

\section{CBP}

CBP is currently the leading cause of disability around the world (7). The cost of medical treatments and loss of work secondary to CBP is estimated to be over $\$ 100$ billion per year, with two-thirds related to lost earnings and diminished productivity (8). The social and economic toll is tremendous, underscoring the importance of understanding the pathophysiology of chronic pain and development of cost-effective management treatments. Further, identifying patients that may benefit from certain interventions can have a dramatic impact on society.

Patients suffering from CBP have been shown to have altered functional connectivity, both during rest and during periods of stimulation. Patients have been found to have significantly altered functional connectivity within the DMN, with significant differences in connectivity not only during rest, but also when performing simple tasks (9). Decreased neural deactivations in the mPFC were specifically noted while performing a simple task, in comparison to healthy controls, suggesting that deactivation in pain-related regions, as occurs in healthy controls, is not exhibited in patients with CBP. The oscillatory dynamics of BOLD signaling in CBP patients in relation to connectivity alterations within the DMN has also been examined, revealing increased high-frequency BOLD oscillations in CBP patients localized to the MPFC, and to a lesser extent in the PCC and bilateral parietal cortex (Fig. 2) (10). The mPFC was also found to demonstrate increased correlation in connectivity to the ACC, INS, and secondary somatosensory cortex, all of which are known to receive nociceptive input from the periphery and are involved in pain perception and modulation. Thus, the increase in high-frequency BOLD oscillations in the $\mathrm{MPFC}$ in CBP patients may be associated with increased functional connectivity, with multiple brain regions involved in pain processing.

Resting-state functional connectivity in the periaqueductal gray (PAG), a region critical to pain modula- 
tion, has also been reviewed, comparing the differences in connectivity in patients with CBP and healthy controls (11). The connectivity between the PAG and mPFC, as well as the PAG and ACC, had enhanced connectivity in patients with chronic back pain, which decreases during pain-inducing maneuvers. The duration of chronic low back pain has also been demonstrated to be negatively correlated with connectivity between the PAG and the INS and the PAG and amygdala. As areas directly related to pain perception and modulation, the INS and amygdala have increased functional connectivity to the PAG early in the development of low back pain, which tapers as illness progresses. These findings indicate that one of the brain's primary pain modulation mechanisms may weaken over time with chronic stimulation. The transition from subacute to chronic back pain has been examined as well (12). Patients with no previous history of back pain were followed for one year after they experienced an episode of subacute back pain, and functional connectivity differences between the patients that began recovering and those whose pain persisted were compared. The nucleus accumbens was identified as having significant differences in the connectivity between the patients, specifically with greater connectivity between the nucleus accumbens and the mPFC and the basal ganglia (BG) noted, indicating potentially another site of disrupted pain modulation.

Functional imaging studies in CBP may have implications for treatment as well. One study analyzed data from a double-blinded trial, which included 30 patients with CBP treated with topical analgesia or a placebo patch. They found that patients with greater connectivity between regions associated with pain, anxiety, fear, and emotional self-reflection, such as the MPFC, have a decreased likelihood of responding to placebo (13). It has also been shown that areas of abnormal connectivity associated with CBP could resolve following treatment (14). Acupunture has also been shown to alter the DMN in patients with $C B P$, correlating with pain relief (15).

\section{Fibromyalgia}

Fibromyalgia affects approximately $2-8 \%$ of the population depending on the diagnostic criteria utilized (16). It is a disease which is associated with significant mental and physical suffering, and many of these patients present with a constellation of symptoms that cannot be explained by conventional physical examination, imaging, or blood work. fMRI has identified distinctive alterations within the neural circuitry of pa- tients suffering from fibromyalgia, leading to potential diagnostic and therapeutic implications.

There are major alterations in the resting-state functional connectivity in patients diagnosed with fibromyalgia when compared to controls (17). Studies have found that the ACC, BG, and sensorimotor cortices all show increased resting-state functional connectivity, with the ACC having increased connectivity with both the BG and INS. There are multiple areas of decreased activity as well, notably the thalamus has decreased connectivity with the INS and PAG, and the ACC and PAG also have decreased connectivity when compared to healthy controls. The abnormalities in the PAG may implicate dysfunction in descending pain modulatory systems as an important component of the mechanism underlying chronic pain in these patients.

Age-related gray matter differences with differing patterns of connectivity have also been noted in individuals with fibromyalgia (18). Significant differences were seen in the overall volume of gray matter, with patients over the age of 50 having less gray matter and those younger than 50 having more gray matter when compared to healthy controls. With the older patients, decreased gray matter was specifically noted in the ACC, mPFC, right premotor cortex, ventrolateral prefrontal cortex, right dorsalateral prefrontal cortex (DLPFC), and right PCC. Younger patients were found to have increased gray matter in the INS, BG, and mPFC. Further, when undergoing pressure stimuli, those with the least amount of gray matter in the PCC were found to be the most sensitive. Young patients also displayed increased resting-state connectivity between the nucleus accumbnes and DLPFC, areas involved in motivation and important pain inhibitory regions, respectively. Additionally, the anterior INS in younger patients displayed decreased resting correlation with the ACC, an area which processes unpleasant emotions associated with painful stimuli. It has been theorized that increased gray matter in areas of pain modulation can reflect an adaptation which allows for normal perception of pressure stimuli in younger patients with fibromyalgia.

Therapies for fibromyalgia have also been evaluated with functional imaging. Transcranial direct current stimulation (tDCS) is one therapy which has emerged to potentially treat fibromyalgia. Patients with fibromyalgia have been examined with functional imaging at baseline and then 5 days after both a real and false tDCS treatment (19). They observed that decreased functional connectivity between regions including 
the thalamus and posterior INS correlated with clinical reductions in pain after both real and false treatment, and the functional connectivity changes last far beyond placebo effect. Another study showed that administering milnacipran to patients with fibromyalgia was associated with decreased connectivity between the ACC and INS, as well as the PAG and the INS (20). Dysfunctional resting-state functional connectivity patterns have also been shown to normalize after physical therapy programs (21). These results suggest that fMRI may prove important for the assessment of treatment responses in patients with fibromyalgia.

\section{CRPS}

CRPS is a debilitating disorder characterized by dysfunction in the autonomic, sensory, and motor systems. Functional imaging has been utilized to examine the connectivity alterations that occur in patients who suffer from this condition. Several specific alterations have been noted, including diminished overall connectivity with the DMN and the frontal cortex, increases in neuronal activity in the ventromedial prefrontal cortex and the inferior parietal lobule (IPL) of the DMN, and within the sensorimotor cortex more diffuse functional connectivity to the cingulate cortices, parietal cortices, temporal cortex, frontal cortex, and thalamus (22).

An association between functional connectivity and clinical symptoms has also been established. When patients reported greater pain intensity, as quantified by the McGill Pain Questionnaire (MPQ), there was a trend towards an increase in functional connectivity of the PCC with the sensorimotor cortex, prefrontal cortex, posterior parietal cortex, IPL, thalamus, and ACC. There was also a negative correlation between pain intensity and functional connectivity of the sensorimotor cortex with the dorsolateral prefrontal cortex and the posterior INS.

Pediatric patients with CRPS have a higher remission rate, which allows for study of connectivity before and after resolution of symptoms (23). In one study, pediatric patients with CRPS were imaged both during the period of symptoms and after recovery. Cold tolerance was measured in the region of pain and its corresponding area in the unaffected limb, and 9 regions of interest were identified to study: the amygdala, caudate, pallidum, putamen, thalamus, ACC, INS, somatosensory cortex, and parietal cortex. Of the regions examined, increased functional connectivity was noted within and between all the regions of interest during cold stimulation; there was also no cold-induced reductions in functional connectivity on the affected side. This finding suggests pain leads to an increased degree of BOLD synchronization within pain processing regions. Furthermore, 5 of the 9 regions of interest (amygdala, anterior cingulate cortex, caudate, postcentral gyrus, and putamen) displayed significantly increased elevations in functional connectivity when cold was applied to the affected limb as compared to cold stimulation of the unaffected limb.

When identifying whether functional connectivity was increased only during the periods of pain or if it was present after resolution of symptoms it was found that only the anterior cingulate showed a transient increase in connectivity, all others (amygdala, caudate, putamen, and postcentral gyrus) displayed distinct areas of persistent increased connectivity. Another study examining pediatric patients with CRPS found overall reduction in functional connectivity of the habenula with multiple brain regions involved in pain modulation, motor function, and cognitive processes, specifically between the anterior midcingulate cortex, DLPFC, motor cortex, and premotor cortex (24).

CRPS treatment is complex and involves a multimodal approach of rehabilitation and medication management, as well as interventional procedures; functional imaging has examined the functional alterations induced by these treatment modalities. Cortical connectivity changes have been seen following treatment with spinal cord stimulation (25). After patients with CRPS underwent spinal cord stimulator implantation they were followed with functional imaging. Significant differences in resting-state connectivity were noted when the patient was in an optimal pain reduction state, including decreased connection between the somatosenory and limbic system. These results suggest that pain may play a significant role in the generation and maintenance of CRPS-associated cortical changes and show the potential for effective assessment of cortical response to various treatments for CRPS patients.

\section{Diabetic Neuropathic Pain}

Diabetic neuropathy continues to be a common cause of neuropathic pain in individuals with diabetes. Beyond pain, diabetic neuropathy leads to several comorbidities, including the formation of lower extremity ulcers (26). The lifelong incidence of foot ulcers in these patients can be as high as $25 \%$ (27). Despite this, evidence suggests that appropriate screening and initiation of treatment can reduce this number and have a significant impact on long-term consequences of the 
disease (28). Utilization of fMRI to assess screening methods and evaluate treatments could enhance outcomes and guide the therapies provided.

In patients with diabetic neuropathy compared to healthy controls, the resting-state profile of the DMN is altered significantly (29). Decreased connectivity between the DMN and regions including the primary sensorimotor area, ACC, inferior temporal cortices, and inferior occipital cortices are observed. Conversely, there is increased connectivity in the left DLPFC, thalamus, INS, inferior parietal lobe, and precuneus. Independent of the peripheral sensory input, these interactions may alter the perceptual correlates of pain, as seen by the decrease in connectivity with the sensorimotor areas and the increase in connectivity between the frontal regions.

Resting-state functional connectivity between the thalamus and the primary somatosensory cortex has also been examined in this patient population (30). A decreased resting-state thalamo-cortical connectivity, specifically between the ventral posterior thalamic nucleus and the sensorimotor, frontal, cingulate, parietal cortices, and the medial dorsal thalamic nucleus, has been noted. Previous studies have suggested a prominent role for the thalamus in pain modulation (31). More broadly, these findings suggest that chronic pain can alter thalamocortical connections and disrupt thalamic feedback.

Attention systems may play a role with pain modulation in patients with diabetic neuropathy. An analysis by Cauda et al (32) of resting-state functional connectivity between the ventral attention network (VAN), dorsal attention network (DAN), and ACC revealed a reduction in resting-state connectivity between these regions (VAN, DAN) when compared to healthy controls. Their investigation theorized that signals induced from chronic pain disrupt the connectivity of attention networks, causing impaired attention processing. Further analysis revealed a general reduction in connectivity between all networks and the dorsal anterior cingulate cortex (dACC), a region typically correlated with attention processes. The authors hypothesize that the dACC plays a crucial role in attention network modulation and that chronic pain may modulate reduced activation of the parieto-fronto-cingulate network through disruption of normal dACC functional connectivity.

Severe chronic pain is often treated with multiple modalities in combination with interventional pain management. In patients with severe chronic pain, a novel therapeutic approach includes training the patient to control activity in relevant parts of the brain and change sensitivity to pain stimuli by voluntary control of brain activity. The fMRI in real time can be used as a feedback instrument (33).

Other applications have provided additional clinical relevance. Haller et al (34) found that real-time fMRI may help in the reduction of chronic tinnitus by neurofeedback from fMRI. Baliki et al (9) found that the cortical regions with exaggerated functional connectivity in patients with chronic back pain was related to emotions, and Rodrigues-Raccke et al (35) have demonstrated that gray matter atrophy in patients with chronic low back pain is reversible. Controlling emotions with neurofeedback from fMRI could potentially be useful in order to decrease exaggerated functional brain activity and reverse the loss of gray matter in patients with chronic back pain.

\section{Discussion}

Chronic pain continues to be one of the greatest challenges facing patients and physicians in medicine today. Not only is there a lack of objective markers for diagnosing and evaluating pain, many of these conditions are difficult to treat. Currently, evaluation of pain is primarily based on self-reported pain scores, which lack objectivity. The validity of such scores is far from ideal, and the Initiative on Methods, Measurement, and Pain Assessment in Clinical Trials has provided recommendations and reviews to guide the further evaluation of pain and treatments of pain through clinical trials with multiple consensus reviews (3). Many conditions that are known to cause pain can be identified radiographically, and physical examination will always be the cornerstone of diagnosis. However, radiologic abnormalities often do not correlate with the patient's symptomatology or severity of pain, complicating the clinical picture.

fMRI, specifically functional connectivity, is a modality which may provide an objective measure of pain. This review focused on functional connectivity changes in patients with chronic pain, specifically examining 4 common chronic pain conditions: CBP, fibromyalgia, CRPS, and diabetic neuropathy. In all 4 conditions, significant alterations in the resting-state networks of the brain have been identified. Identifying such alterations may aid in diagnosis and further clarify potential central mechanisms of pain. Perhaps more importantly, pre- and post-examinations of functional connectivity may provide an objective marker for treatment efficacy, particularly when combined with self-reported improvements in pain. 


\section{Limitations}

In general, the studies included within this topical review were cross-sectionals and randomized controlled trials. Limitations of these studies included small sample sizes and the observational nature of some of them. At this stage, studies with larger sample sizes and long-term follow-up are required to understand the changes in connectivity for these chronic pain states over a period of time.

\section{Conclusion}

The potential for using functional imaging in diagnosis and monitoring of conditions is both challenging and ambitious. As alterations in functional connectivity are uncovered, these findings will need to be corroborated with clinical data including physical examinations, radiological imaging, and laboratory tests. Access and current feasibility of $\mathrm{fMRI}$ is limited due to its specialization and complexity; however, the potential is tremendous. Functional connectivity has already improved the understanding of mechanisms underlying pain perception and modulation. In summary, fMRI is an exciting new frontier that may further the advancement of pain medicine. Patients with chronic pain exhibit significant structural and functional neuronal change, which appear specific to various pain conditions. Thus, the use of $\mathrm{fMRI}$ may contribute in establishing a better understanding of these pain conditions, resulting in improved treatments and better quality of life for those afflicted with chronic pain syndromes.

In conclusion, neuroimaging and functional connectivity have the potential to usher in a new era of pain medicine that may enhance diagnosis of painful disease states and improve assessment of therapies. The non-invasive technology of real-time feedback of brain activity as related to pain could potentially enable patients with severe chronic pain to voluntarily control functional connectivity-producing pain relief and training to the brain to alter brain function by modulation. More research is warranted regarding real-time fMRI (rtfMRI). There are still questions about its usefulness in the diagnosis of chronic pain syndromes and its ability to assess therapy after interventional pain management. rtfMRI may also prove beneficial in the study of reversibility of gray matter loss, neural plasticity, and improvement of emotions.

\section{References}

1. Global Burden of Disease Study 2013 Collaborators. Global, regional, and national incidence, prevalence, and years lived with disability for 301 acute and chronic diseases and injuries in 188 countries, 1990-2013: A systematic analysis for the Global Burden of Disease Study 2013. Lancet 2015; 386:743-800.

2. Institute of Medicine (US) Committee on Advancing Pain Research, Care, and Education. Relieving pain in America: A blueprint for transforming prevention, care, education, and research. Washington (DC): National Academies Press; 2011.

3. Edwards RR, Dworkin RH, Turk DC, Angst MS, Dionne R, Freeman R, Hansson $\mathrm{P}$, Haroutounian $\mathrm{S}$, Arendt-Nielsen L, Attal N, Baron R, Brell J, Bujanover S, Burke LB, Carr D, Chappell AS, Cowan P, Etropolski M, Fillingim RB, Gewandter JS, Katz NP, Kopecky EA, Markman JD, Nomikos G, Porter L, Rappaport BA, Rice AS, Scavone JM, Scholz J, Simon LS, Smith SM, Tobias J, Tockarshewsky T, Veasley C, Versavel M, Wasan AD, Wen W, Yarnitsky D. Patient phenotyping in clinical trials of chronic pain treatments.
Pain 2016; 157:1851-1871.

4. Apkarian AV, Bushnell MC, Treede RD, Zubieta JK. Human brain mechanisms of pain perception and regulation in health and disease. Eur J Pain 2005; 9:463-484.

5. Chapin H, Bagarinao E, Mackey S. Realtime $\mathrm{fMRI}$ applied to pain management. Neurosci Lett 2012; 520:174-181.

6. Friston KJ. Functional and effective connectivity: A review. Brain Connect 2011; 1:13-36.

7. Buchbinder R, Blyth FM, March LM, Brooks P, Woolf AD, Hoy DG. Placing the global burden of low back pain in context. Best Pract Res Clin Rheumatol 2013; 27:575-589.

8. Katz JN. Lumbar disc disorders and lowback pain: Socioeconomic factors and consequences. J Bone Joint Surg Am 2006; 88:21-24.

9. Baliki MN, Geha PY, Apkarian AV, Chialvo DR. Beyond feeling: Chronic pain hurts the brain, disrupting the defaultmode network dynamics. J Neurosci 2008; 28:1398-1403.

10. Baliki MN, Baria AT, Apkarian AV. The cortical rhythms of chronic back pain. J Neurosci 2011; 31:13981-13990.

11. Yu R, Gollub RL, Spaeth R, Napadow V, Wasan A, Kong J. Disrupted functional connectivity of the periaqueductal gray in chronic low back pain. Neuroimage Clin 2014; 6:100-108.

12. Baliki MN, Petre B, Torbey S, Herrmann KM, Huang L, Schnitzer TJ, Fields HL, Apkarian AV. Corticostriatal functional connectivity predicts transition to chronic back pain. Nat Neurosci 2012; 15:1117-1119.

13. Hashmi JA, Baria AT, Baliki MN, Huang L, Schnitzer TJ, Apkarian VA. Brain networks predicting placebo analgesia in a clinical trial for chronic back pain. Pain 2012; 153:2393-2402.

14. Deko M, Shir Y, Ouellet JA, Ware MA, Stone LS, Seminowicz DA. Partial recovery of abnormal insula and dorsolateral prefrontal connectivity to cognitive networks in chronic low back pain after treatment. Hum Brain Mapp 2015; 36:2075-2092.

15. Li J, Zhang JH, Yi T, Tang WJ, Wang SW, 
Dong JC. Acupuncture treatment of chronic low back pain reverses an abnormal brain default mode network in correlation with clinical pain relief. Acupunct Med 2013; 32:102-108.

16. Clauw DJ. Fibromyalgia: A clinical review. JAMA 2014; 311:1547-1555.

17. Cifre I, Sitges C, Fraiman D, Muñoz MÁ, Balenzuela $P$, González-Roldán A, Martínez-Jauand M, Birbaumer N, Chialvo DR, Montoya P. Disrupted functional connectivity of the pain network in fibromyalgia. Psychosom Med 2011; 74:55-62.

18. Ceko M, Bushnell MC, Fitzcharles MA, Schweinhardt P. Fibromyalgia interacts with age to change the brain. Neuroimage Clin 2013; 3:249-26o.

19. Cummiford CM, Nascimento TD, Foerster BR, Clauw DJ, Zubieta JK, Harris $\mathrm{RE}$, DaSilva AF. Changes in resting state functional connectivity after repetitive transcranial direct current stimulation applied to motor cortex in fibromyalgia patients. Arthritis Res Ther 2016; 18:40.

20. Schmidt-Wilcke T, Ichesco E, Hampson J, Kairys A, Peltier S, Harte S, Clauw D), Harris RE. Resting state connectivity correlates with drug and placebo response in fibromyalgia patients. Neuroimage Clin 2014; 6:252-261.

21. Flodin P, Martinsen S, Mannerkorpi K, Löfgren M, Bileviciute-Ljungar I, Kosek E, Fransson P. Normalization of aberrant resting state functional connectivity in fibromyalgia patients following a three month physical exercise therapy. Neuroimage Clin 2015; 9:134-139.
22. Bolwerk A, Seifert F, Maihöfner C. Altered resting-state functional connectivity in complex regional pain syndrome. J Pain 2013; 14:1107-1115.

23. Linnman C, Becerra L, Lebel A, Berde C, Grant PE, Borsook D. Transient and persistent pain induced connectivity alterations in pediatric complex regional pain syndrome. PLoS One 2013; 8:e57205.

24. Erpelding N, Sava S, Simons LE, Lebel A, Serrano P, Becerra L, Borsook D. Habenula functional resting-state connectivity in pediatric CRPS. J Neurophysio 2013; 111:239-247.

25. Deogaonkar $M$, Sharma $M$, Oluigbo C, Nielson DM, Yang X, Vera-Portocarrero L, Molnar GF, Abduljalil A, Sederberg PB, Knopp M, Rezai AR. Spinal cord stimulation (SCS) and functional magnetic resonance imaging (fMRI): Modulation of cortical connectivity with therapeutic SCS. Neuromodulation 2015; 19:142-153.

26. Reiber GE, Vileikyte L, Boyko EJ, del Aguila M, Smith DG, Lavery LA, Boulton AJ. Causal pathways for incident lowerextremity ulcers in patients with diabetes from two settings. Diabetes Care 1999; 22:157-162.

27. Boulton AJ, Vileikyte L, Ragnarson-Tennvall G, Apelqvist J. The global burden of diabetic foot disease. Lancet 2005; 366:1719-1724.

28. Singh N, Armstrong DG, Lipsky BA. Preventing foot ulcers in patients with diabetes. JAMA 2005; 293:217-228.

29. Cauda F, Sacco K, Duca S, Cocito D,
D’Agata F, Geminiani GC, Canavero S. Altered resting state in diabetic neuropathic pain. PLoS One 2009; 4:e4542.

30. Cauda F, Sacco K, D’Agata F, Duca S, Cocito D, Geminiani G, Migliorati F, Isoardo G. Low-frequency BOLD fluctuations demonstrate altered thalamocortical connectivity in diabetic neuropathic pain. BMC Neurosci 2009; 10:138.

31. Garcia-Larrea L, Maarrawi J, Peyron R, Costes N, Mertens P, Magnin M, Laurent $\mathrm{B}$. On the relation between sensory deafferentation, pain and thalamic activity in Wallenberg's syndrome: A PETscan study before and after motor cortex stimulation. Eur J Pain 2006; 10:677-688.

32. Cauda F, D'Agata F, Sacco K, Duca S, Cocito D, Paolasso I, Isoardo G, Geminiani G. Altered resting state attentional networks in diabetic neuropathic pain. J Neurol Neurosurg Psychiatry 2009; 81:806-811.

33. deCharms RC, Maeda F, Glover GH, Ludlow D, Pauly JM, Soneji D, Gabrieli JDE, Mackey SC. Control over brain activation and pain learned by using realtime functional MRI. Proc Natl Acad Sci USA 2005; 102:18626-18631.

34. Haller S, Birbaumer N, Veit R. Realtime $\mathrm{fMRI}$ feedback training may improve chronic tinnitis. Eur Radiol 2010; 20:696-703.

35. Rodriguez-Raecke R, Niemeier A, Ihle $\mathrm{K}$, Ruether $\mathrm{W}$, May A. Brain gray matter decrease in chronic pain is the consequence and not the cause of pain. J Neurosci 2009; 29:13746-13750. 